\title{
La vinculación como potenciadora de la formación profesional de profesionales de ingeniería: Propuesta de acciones con base en experiencias en la Universidad Autónoma de Nuevo León
}

\author{
The Concept of Connection as an Enhancer of the Professional Training of the Engineer: \\ Experiences at Universidad Autónoma De Nuevo León
}

\section{A vinculação como potenciadora da formação profissional de profissionais de engenharia: Proposta de ações com base em experiências na Universidade Autônoma de Novo León}

Jaime Arturo Castillo-Elizondo Universidad Autónoma de Nuevo León Monterrey, México jaime.castilloe@uanl.mx

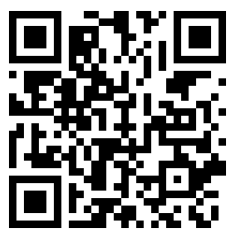

http://orcid.org/0000-0003-2100-3115

Nivia Tomasa Álvarez-Aguilar Universidad Autónoma de Nuevo León, Monterrey, México nivial12@yahoo.es

https://orcid.org/0000-0003-4110-8862

Arnulfo Treviño-Cubero Universidad Autónoma de Nuevo León

Monterrey, México arnulfotrevinoc@uanl.mx http://orcid.org/0000-0002-0958-8352

Recibido • Received • Recebido: 12 / 03 / 2018

Corregido • Revised • Revisado: 20 / 10 / 2018

Aceptado • Accepted • Aprovado: 15/ 01 / 2019

\begin{abstract}
Resumen: La vinculación de la universidad con el entorno es un tema siempre actual y de continuo perfeccionamiento. En el caso de profesionales en ingeniería se constituye en un imperativo para el logro de una adecuada formación. El objetivo fundamental de este estudio consiste en valorar las posibilidades de la vinculación en las escuelas y facultades de ingeniería para la formación de profesionales que se adapten y, a la vez, contribuyan a transformar su entorno con un sentido ético y responsable. Se utilizó un diseño no experimental de tipo descriptivo de corte longitudinal que permitió identificar aquellos vacíos que requieren ser llenados desde una perspectiva teórica como sustento de las acciones prácticas descritas. Los sujetos participantes fueron estudiantes de últimos semestres de 10 carreras de ingeniería. Se analiza críticamente la poca presencia del enfoque integral de la vinculación en las fuentes consultadas. A partir de aquí, se determinaron una serie de principios para la vinculación con el entorno. Se presenta un resultado concluyente de la primera fase de la
\end{abstract}


doi: http://dx.doi.org/10.15359/ree.23-2.11

URL: http://www.una.ac.cr/educare

CORREO: educare@una.cr

investigación que describe acciones de vinculación orientadas al desarrollo profesional estudiantil, cuyos resultados se medirán en una próxima etapa. Estas propuestas constituyen un referente desde su concepción, funciones y propósitos: Las condiciones, formas y métodos deberán adaptarse al contexto. Se concluye que la vinculación de la universidad con el entorno carece de un enfoque integral y de una sistematización e innovación constante, además de que en las facultades existen múltiples posibilidades para la innovación de nuevas vías para la formación profesional en ingeniería.

Palabras claves: Vinculación; formación profesional; estudiante de ingeniería.

Abstract: The connection between the university and the environment is a constantly updated topic. In the case of the engineering is necessary for the achievement of adequate training. The main objective of this study is to assess the possibilities of linking engineering schools and faculties to train professionals who adapt and, at the same time, contribute to transform their environment ethically and responsibly. A non-experimental descriptive design was used to identify those gaps that need to be filled from a theoretical perspective as a basis for the practical actions described. The participants were students of last semesters of 11 engineering careers. The limited presence of the integral approach of that connection is critically analyzed in the consulted sources. From this point, a series of principles for the connection with the environment were determined. A conclusive result of the first phase of the research is presented. This result describes linking actions oriented to the professional development of the students, whose results will be measured in a next stage. These proposals constitute a reference from its conception, functions, and purposes: the conditions, forms, and methods must be adapted to the context. It is concluded that the link between the university and the environment lacks an integral approach and systematization, and constant innovation. It is also concluded that, in the faculties, there are multiple possibilities for the innovation of new avenues for the professional training of the engineer.

Keywords: Linking; professional training; engineering student.

Resumo: A vinculação da universidade com seu redor é um tema sempre atual e de contínuo aperfeiçoamento. No caso de profissionais de engenharia, é imperativo obter uma adequada formação. O principal objetivo deste estudo é avaliar as possibilidades de articulação em escolas e faculdades de engenharia para a formação de profissionais que se adaptem e, ao mesmo tempo, contribuam para transformar seu redor com um sentido ético e responsável. Utilizou-se um desenho não experimental de tipo descritivo de corte longitudinal que permitiu identificar as lacunas que precisam ser preenchidas do ponto de vista teórico como base para as ações práticas descritas. Os participantes foram estudantes dos últimos semestres de 10 carreiras de engenharia. Analisa-se criticamente a pouca presença do enfoque integral da vinculação em as fontes consultadas. A partir de aqui, determinaram-se uma série de princípios para a vinculação com o redor. Apresenta-se um resultado conclusivo da primeira fase da investigação que descreve ações de vinculação orientadas ao desenvolvimento profissional do estudante, cujos resultados serão medidos em uma próxima etapa. Estas propostas constituem uma referência desde a sua concepção, funções e propósitos: As condições, formas e métodos devem ser adaptados ao contexto. Conclui-se que o vínculo entre a universidade e o redor carece de um enfoque integral e de uma sistematização e inovação constante, além de que nas faculdades existem múltiplas possibilidades para a inovação de novas vias para a formação profissional em engenharia.

Palavras chaves: Vinculação; formação profissional; estudante de engenheira. 


\section{Introducción}

Cada día el mundo experimenta nuevos cambios en todas las esferas de la actividad, por ello, generalmente, la universidad social no puede ofrecer una preparación que satisfaga las necesidades sociales. Como apunta (Palma, 2012) los grupos profesionales en ingeniería, una vez graduados, se encuentran con una realidad muy demandante que no fue tomada en cuenta por el plan de estudios en su tiempo en la facultad.

La Organización para la Cooperación y el Desarrollo Económico (OCDE), en las últimas décadas, ha otorgado un rol importante a la educación superior, impulsando iniciativas en el modelo de universidad relacionadas con la vinculación entre la universidad, el gobierno y la empresa, así como en procesos de evaluación y rendición de cuentas para atender, entre otras problemáticas, la relacionada con la vinculación y su pertinencia social.

La capacidad de las universidades para afrontar los grandes cambios de la actualidad y aprovechar las oportunidades que presentan las nuevas circunstancias de su entorno, se incrementará en la medida en que dispongan de una infraestructura adecuada y recursos suficientes para subsidiar su desarrollo institucional y poder cumplir con el compromiso social que las caracteriza y así, ser reconocidas por la comunidad a la que sirven. (Alcántar y Arcos, 2009, p. 6)

Para el logro de este propósito, la vinculación con el entorno juega un rol fundamental, puesto que constituye una vía importante para obtener recursos. Esto es posible siempre y cuando sea una interacción donde todos los factores implicados se vean favorecidos: universidad, empresas, instituciones sociales y gobierno.

Esactual el planteamiento de Camposy Sánchez(2005)cuando sostienen quela universidad latinoamericana no ha logrado desarrollar la vinculación deseada con sus respectivos entornos productivos.

A pesar de que en el discurso se reconozca la importancia de la vinculación para los diferentes factores que en ella intervienen, como señalan López- Parada y Martínez-Dajui (2014, p. 2):

La vinculación entre la universidad y sus respectivos entornos productivos es una tarea pendiente de realizar. Existen evidencias solidas de que se inicia, pero no tiene la amplitud que desearían aquellos que sólo manejan el modelo norteamericano o francés, en el que algunas universidades operan con altos presupuestos derivados de la vinculación con empresas o fundaciones. 
doi: http://dx.doi.org/10.15359/ree.23-2.11

URL: http://www.una.ac.cr/educare

CORREO: educare@una.cr

En esa misma línea de pensamiento se coincide con Pérez (2016, p. 5) cuando enfatiza:

A pesar dela importanciay los beneficiosquegenera la vinculación de las IES con las empresas en la sociedad este tipo de cooperación aún es muy escasa en países latinoamericanos, lo cual ha causado una disminución en el valor que se le da a dicha actividad, así como también ha mermado la iniciativa de cooperar en este tipo de proyectos.

No obstante a lo antes expresado, las facultades de ingeniería promueven acciones de manera paralela a la formación académica para la rápida y eficaz inserción de su estudiantado egresado al mercado laboral, entre ellas ocupan un lugar destacado las actividades donde se implican directamente estudiantes. No obstante, se ha de considerar que, debido a los rápidos y constantes cambios en la industria, la vinculación requiere de una renovación sistemática de su concepción y desarrollo.

Aunque en los últimos años se ha fortalecido considerablemente esta actividad, SarabiaAltamirano (2016, p. 20) acota:

En México, las interacciones universidad-empresa han sido frágiles e irregulares, en gran medida por la limitada vinculación, así como la baja generación, diseminación y absorción de conocimiento tecnológico. El desajuste entre el suministro de conocimiento y la demanda de las empresas surgió desde el origen.

Lo anterior indica que el problema de la vinculación de los centros de educación superior con el entorno es complejo y demanda una actualización sistemática acorde con las exigencias del desarrollo socioeconómico. Del mismo modo, depende en gran medida de los modelos de gestión que se asuman por estas instituciones. En este sentido, es necesario aplicar métodos novedosos puesto que, como destaca Pertuz (2014), la organización que adopten las instituciones debe estar acorde con las estructuras innovadoras que se requieren en la actualidad.

Lógicamente, la solución a los problemas que enfrenta la vinculación requiere, ante todo, de una concientización de los diversos factores que de una u otra forma impactan en la formación del estudiantado en ingeniería que la sociedad necesita. Relacionado con esto, Bautista (2014, p. 123) señala: "solamente cuando las empresas mexicanas se apoyen en las universidades y el gobierno, serán capaces de competir con cualquier mercado del exterior".

\section{Objetivo general}

El objetivo fundamental de este estudio consiste valorar las posibilidades de la vinculación en las escuelas y facultades de ingeniería para la formación de profesionales que se adapten 
y, a la vez, contribuyan a transformar su entorno con un sentido ético y responsable. Para el logro de este objetivo fue necesario el cumplimiento de objetivos específicos relacionados con el análisis crítico de la bibliografía sobre el tema objeto de estudio para identificar principios esenciales que han de sustentar la vinculación.

Esta investigación se realiza a la luz de las exigencias de la sociedad actual para las personas profesionales de la ingeniería. Por ello, se parte de un análisis crítico de la problemática abordada destacando aspectos positivos pero también identificando aquellos vacíos que requieren ser llenados desde una base teórica que sustente las acciones prácticas. Para cumplir con este objetivo se describen algunas experiencias que, de acuerdo con su concepción y propósitos, impactan favorablemente en la formación del estudiantado de ingeniería, además pueden ser utilizados en otras facultades y escuelas en correspondencia con las características propias y del contexto.

El trabajo está estructurado del modo siguiente: En la introducción se explica la importancia, actualidad y pertinencia del tema lo que determinó el objetivo general del estudio realizado. Seguidamente se exponen los antecedentes que permitieron identificar los aspectos más relevantes relacionados con el objeto de estudio, así como aquellas áreas de oportunidades que requieren ser tomadas en cuenta. A partir de este análisis, se revela la justificación. Se especifican los conceptos fundamentales y relaciones que componen los sustentos teóricos.

Posteriormente se detalla la metodología usada para dar cumplimiento al objetivo propuesto y finalmente se describen diferentes acciones basadas en experiencias de la práctica de la vinculación que conjuntamente con el análisis crítico de las fuentes relacionadas con el tema, reflejan las potencialidades del proceso de vinculación para una formación profesional en ingeniería acorde a las necesidades de la sociedad actual.

\section{Antecedentes y justificación del estudio}

En los últimos tiempos se observa un esfuerzo de las universidades para enfocarse tanto en la formación del ser humano en los aspectos personales de actitudes y valores como en cuanto a conocimientos y competencias profesionales, ambas dimensiones marcan la calidad educativa de una institución. Para favorecer los procesos formativos mencionados, se demanda de grandes recursos, por lo que es muy importante la búsqueda y puesta en marcha de acciones tendientes al logro de una gestión más eficaz del proceso de vinculación.

Tal y como expresan Soto et al. (2007):

La vinculación puede ser un aspecto estratégico para la formación de los recursos humanos que se insertan al campo laboral y también puede impactar en el diseño curricular y la elaboración de planes y programas de estudio que realizan las universidades para que respondan a las necesidades de la sociedad [contemporánea]. (pp. 3-4) 
doi: http://dx.doi.org/10.15359/ree.23-2.11

URL: http://www.una.ac.cr/educare

CORREO: educare@una.cr

No obstante, en ocasiones se enfoca el tema de la vinculación solo en el aspecto económico, así por ejemplo para Jiménez-Montecinos (2016) "el llamado a las universidades es empujar ligeramente la balanza hacia un entorno que facilite la interacción con la industria y apueste por la innovación y la generación de nuevos sectores con potencial económico" (p. 10). Este autor presta atención de manera casi exclusiva al aspecto económico y a la innovación.

Diferentes estudios (D'Este, Castroy Molas Gallart, 2014) identifican la vinculación como una tercera misión de la universidad en relación con las dos primeras: la docencia y la investigación. Argumentan que está recibiendo una gran atención debido a los cambios que están ocurriendo en las relaciones CTS, así como por el impacto de la producción del conocimiento en la sociedad en general.

En las diversas posiciones acerca de la vinculación "prevalece la idea de que [su esencia radica en] las relaciones que existen -o deben existir- entre la universidad y la sociedad de las cuales forma parte" (Campos y Sánchez, 2005, p. 4). También aparece con mucha frecuencia la idea de considerar la vinculación como una función deseable e imprescindible desde los mismos orígenes de las instituciones de educación superior (Campos y Sánchez, 2005).

Una limitación importante en cuanto al impacto formativo en el estudiantado de ingeniería está en que muchas veces se limita a la vinculación universidad-empresa. Así lo acota Ibarra-Colado (2008) en la reseña realizada al libro La vinculación universidad-empresa: Miradas críticas desde la universidad pública, de las autoras Llomovate, Juarros, Naidorf y Guelman, al considerar que la vinculación generalmente se ha visto limitada a su interacción con la empresa; sin embargo, ha obviado diferentes instituciones sociales que pueden ser agentes de cambio en los países de nuestra región

Desdela perspectiva anterior, autoras comoGutiérrez (2004) coinciden en quela vinculación ya no puede ser considerada como en sus inicios, que era una exigencia de las políticas de acción hacia las universidades. Por el contrario, ha pasado a ser un requisito indispensable para el desarrollo tanto económico como científico tecnológico. Del mismo modo, la formación de estudiantes en ingeniería en la época actual no se concibe sin una eficiente vinculación donde se potencie no solo la obtención de recursos materiales, sino también el desarrollo de las personas involucradas en este proceso, en especial el estudiantado.

Enel caso particular de las facultades yescuelas de ingeniería, por sus propias características, se requiere de una renovación constante de las vías y acciones del proceso de vinculación. Producto del acelerado desarrollo científico tecnológico surgen nuevas carreras que demandan saberes de otras disciplinas para poder dar cumplimiento a las nuevas necesidades sociales, como lo es, por ejemplo, la ingeniería biomédica. 
A lo anterior se añade el énfasis que se pone actualmente en el tema de la responsabilidad social:

En los círculos académicos, durante los últimos años, se ha discutido el tema de la responsabilidad social, como un aspecto relevante, particularmente porque brinda una dimensión en la cual las IES desarrollan una toma de conciencia de carácter holista, que involucra a la propia organización de las instituciones y el impacto de estas en su entorno social. (Cantú-Martínez, 2013, p. 43)

De ahí la importancia de la vinculación para la formación de profesionales en ingeniería que estén a tono con la época actual, esta dada entre otras cuestiones, porque como acota (Bautista, 2014):

El conocimiento y la innovación se vislumbran como capaces de generar competencias, destacando tres grandes rubros en la producción y aprendizaje en nuestra era del conocimiento; 1 ) aprender haciendo; 2) aprender usando y; 3 ) aprender interactuando. (pp. 123-124)

Los trabajos sobre este tema acreditan que "son tres los modelos más reconocidos y estudiados que interpretan la relación Estado-Empresa-Universidad" (López, 2014, p. 112). Estos modelos, según expone López (2014), son: “El triángulo científico-tecnológico, también denominado triángulo de Sábato, el Modelo de la Triple Hélice y los Sistemas de Innovación" (p. 112). Este último se está imponiendo, ya que la innovación es propósito esencial de cualquier institución social.

Lo expuesto indica la urgencia de una mayor interacción de las universidades con el entorno. Aunque esto no es algo nuevo, la realidad demuestra que dicha interacción requiere ser reconocida y además renovada. No obstante su larga trayectoria, este tema está necesitado de un mejoramiento profundo.

La limitación antes señalada es una situación que atenta contra la formación de profesionales en la ingeniería actual.

La enseñanza de la ingeniería desde su surgimiento ha estado condicionada por diferentes cambios que la han hecho evolucionar y a la vez enriquecerse. Constituye una preocupación de todos los tiempos, la formación de un ingeniero acorde con las necesidades del entorno en que vive y se desenvuelve y la manera en que debe enfrentar las mismas [sic]. (Capote, Rizo y Bravo, 2016, p. 22) 
doi: http://dx.doi.org/10.15359/ree.23-2.11

URL: http://www.una.ac.cr/educare

CORREO: educare@una.cr

\section{Referentes conceptuales acerca de la vinculación y su impacto en la formación profesional en ingeniería}

Cuando se hace referencia a un desarrollo exitoso de profesionales en ingeniería, una vez que se gradúen, se precisa hacer alusión a su formación profesional, conceptualizada por Fernández (2001) en los siguientes términos:

Conjunto de procesos sociales de preparación y conformación del sujeto, referidos a fines precisos para un posterior desempeño en el ámbito laboral. Además [aclara este autor, identifica dicha formación con], el proceso educativo que tiene lugar en las instituciones de educación superior, orientado a que los alumnos obtengan conocimientos, habilidades, actitudes, valores culturales y éticos, contenidos en un perfil profesional y que corresponda a los requerimientos para un determinado ejercicio de una profesión. (p. 28)

En este mismo sentido, Pacheco (2012) sostiene que la histórica relación entre universidad, conocimiento y sociedad ha estado cuestionada precisamente por las dificultades que presenta en la formación de profesionales capaces de enfrentar las condiciones laborales una vez logren su graduación.

Y es que "precisamente, la formación profesional definida como un sistema de principios, conocimientos, habilidades, decisiones y acciones orientadas al desarrollo de talentos ante una necesidad o estrategia organizacional, alude a estrategias, decisiones, objetivos, razonamientos y evaluaciones" (García, Carreón y Hernández, 2014, p. 109). Significa que esta dimensión de la formación requiere ser rigurosamente planificada y controlada.

Cámara-Zapata (2016) argumenta que"en las últimas tres décadas, el desarrollo tecnológico ha propiciado cambios con repercusiones en multitud de ámbitos, incluidas la formación en ingeniería y su actividad profesional" (p. 121). Y añade:

La universidad debe hacer más visible y cercana la formación en ingeniería para la empresa privada, en especial para las PYMES [pequeñas y medianas empresas]. Es importante desarrollar iniciativas que acerquen la universidad y la empresa, con el objetivo de mejorar su aportación al desarrollo social. (Cámara-Zapata, 2016, p. 121)

Es conveniente agregar, según Herrera (2006), que las insuficiencias en la formación del estudiantado graduado universitario para enfrentar con calidad sus funciones profesionales en las empresas en las que deben desarrollar su vida laboral se derivan ... [en gran medida] de las debilidades de una relación universidad empresa que pueda cumplir con eficiencia y eficacia este cometido (p. 1). 
Es conocido que las diferentes carreras de ingeniería están presentes en cada una de las innovaciones que se realizan en el campo de la tecnología. Sin embargo, la preparación del estudiantado en el ámbito laboral no siempre es la deseada, porque adolece de múltiples competencias que trascienden la formación relacionada con el aspecto técnico. Las diferentes ramas de la ingeniería precisan de especialistas en campos laborales con sus propias características que no son privativas de estas carreras como son: gerencia, administración, investigación, entre otros.

Hoy día el acelerado desarrollo científico tecnológico, aunado a la globalización, como bien apuntan Kindelán y Martín (2008), aumentan las exigencias a profesionales de la ingeniería en cuanto a competencias genéricas que les permitan un desarrollo integral para acometer con eficiencia las altas demandas en su campo laboral y profesional.

Precisamente por lo antes expuesto, la Asociación Iberoamericana de Instituciones de Enseñanza de la Ingeniería (ASIBEI, 2015) ha definido un perfil profesional en ingeniería iberoamericano como:

Un ingeniero [o ingeniera] global con compromiso y pertinencia local, con sólidas bases científicas, técnicas, tecnológicas, culturales, y con arraigados valores y principios, consciente de la importancia y significado de sus nexos con la historia y el desarrollo regional, fiel a sus compromisos sociales y ambientales, atento a la identificación de los problemas y oportunidades del entorno para actuar de manera responsable y competente en cualquier escenario nacional e internacional. (párr.6)

Para lograr el perfil del profesional en ingeniería antes señalado se requiere un enfoque integral en la preparación del estudiantado de ingeniería desde su ingreso a la facultad. De acuerdo con Valera (2010):

La formación del profesional constituye... el proceso en el que los sujetos desarrollan el compromiso social y profesional, la flexibilidad ante la cultura, la trascendencia en su contexto, toda vez que elevan su capacidad para la reflexión divergente y creativa, para la evaluación crítica y autocrítica, para solucionar problemas, tomar decisiones y adaptarse flexiblemente a un mundo cambiante. (p. 119)

Lo anterior se puede constatar por un estudio presentado en el Congreso Word Engineering Education Forum \& Global Engineering Deans Council (WEEF y GEDC, 2016, citado por ASIBEI, 2016), acerca de las habilidades que están exigiendo los entes empleadores. En este análisis participaron los sectores académico, gubernamental, estudiantil e industrial. Se determinaron las habilidades más importantes de acuerdo con estos entes empleadores, para los grupos egresados de ingeniería en los últimos años. 
doi: http://dx.doi.org/10.15359/ree.23-2.11

URL: http://www.una.ac.cr/educare

CORREO: educare@una.cr

En la Tabla 1 aparece el listado de las habilidades de las que adolecen los grupos egresados, según las personas empresarias; estas fueron determinadas por la Encuesta de Competencias Profesionales (Centro de Investigación para el Desarrollo [CIDAC] 2014, p. 20). Se puede observar la prioridad otorgada a las competencias que tienen relación con el desempeño en general de la persona, las que le permiten reorientarse rápidamente en correspondencia con las condiciones cambiantes de la sociedad actual.

Nótese que estas competencias no son privativas de especialistas en ingeniería. Es significativo que las habilidades detectadas responden a un tipo de profesional: versátil, emprendedor, responsable, creativo y con dominio de un idioma extranjero.

Tabla 1: Competencias básicas que no encuentran las empresas

\begin{tabular}{l} 
Competencias \\
Comunicación escrita en español \\
Comunicación oral en español \\
Comunicación oral en inglés \\
Puntualidad \\
Sentido de responsabilidad \\
Iniciativa o proactividad \\
Capacidad de síntesis de información \\
Pensamiento lógico y ágil \\
\hline
\end{tabular}

Nota: Tomado de ENCOP (2014, citado en CIDAC, 2014, p. 20).

La Tabla 2 muestra resultados más recientes en cuanto a las habilidades demandadas a profesionales en ingeniería, pero los resultados son muy similares a los anteriores. Aunque se nota una cierta diferencia en el enunciado, se observa que las habilidades, según se planteó en el (World Economic Forum), poseen un carácter general que permite a los grupos de ingenierías adaptarse a los nuevos y acelerados cambios del mundo empresarial.

Es interesante conocer que los entes empleadores requieran habilidades de carácter general y no específicas del campo de la ingeniería. Esto se puede entender, si reconocemos que los procesos tecnológicos están cambiando de manera acelerada, por lo que las habilidades específicas requeridas son muy variables. Profesionales en ingeniería con un desarrollo integral tendrán la capacidad de adaptarse y renovarse con facilidad. 
Tabla 2. Las habilidades requeridas para profesionales en ingeniería

\section{0 habilidades para desarrollar en ingeniería según (World Economic Forum)}

1. Solución de problemas complejos

2. Trabajo en equipo

3. Manejo de personal

4. Pensamiento crítico

5. Poder de negociación

6. Control de calidad

7. Orientación de servicios

8. Juicio y toma de decisiones

9. Entendimiento activo

10. Creatividad

Nota: Adaptado de ASIBEl (2016, columna 2, en 2015, filas 1 a 10).

Tanto las experiencias obtenidas en la gestión de la vinculación por varios años e investigaciones realizadas, han permitido reconocer que existen múltiples áreas de oportunidades que requieren ser tomadas en cuenta para lograr un enfoque "más integral" $y$ sistemático en la actividad de vinculación en las escuelas y facultades de ingeniería (Castillo, Reynoso, Álvarez y Torres, 2013).

Entre estas áreas de oportunidades se encuentran:

- Insuficiente planificación, seguimiento, sistematización y retroalimentación en la gestión de la vinculación.

- No siempre se involucran todos los factores que pueden impactar en la vinculación, limitándose muchas veces a la interacción facultad-empresas.

- La participación de personal directivo y docente no es lo sufrientemente satisfactoria.

- Es limitada la comprensión del alcancé de la vinculación, se limita en ocasiones a la obtención de recursos y se subvalora su papel formativo.

- En ocasiones la disposición y aporte de las empresas a esta actividad es limitada.

- Se precisa de una gestión de la vinculación más innovadora y creativa, acorde con las condiciones dinámicas y cambiantes del desarrollo científico tecnológico y de la sociedad en general. 
doi: http://dx.doi.org/10.15359/ree.23-2.11

URL: http://www.una.ac.cr/educare

CORREO: educare@una.cr

\section{Metodología}

Se utilizó un diseño no experimental de tipo descriptivo de corte longitudinal que permitió identificar aquellos vacíos que requieren ser llenados desde una perspectiva teórica como sustento de las acciones prácticas descritas. Es conocido que a este tipo de estudio también se le conoce como estudios transversales o longitudinales. Independiente de la clasificación a la que pertenezca "son estudios observacionales, en los cuales no se interviene o manipula el factor de estudio, es decir, se observa lo que ocurre con el fenómeno en estudio en condiciones naturales, en la realidad" (García, 2004, p. 1). Estas características se complementan en este trabajo con un análisis crítico de la bibliografía a través de la elaboración personal de los autores y autoras. En este caso, el fenómeno general sometido a análisis lo constituye el proceso de vinculación en las carreras de ingeniería.

El fenómeno particular estudiado fue el proceso de vinculación en sus diferentes manifestaciones de la Facultad de Ingeniería Eléctrica de la Universidad Autónoma de Nuevo León (UANL) que cuenta con 11 carreras y más de 18000 estudiantes.

Se aplicó el método documental que permitió identificar las principales bases teóricas que sustentan el trabajo, a partir del análisis de diferentes criterios y propuestas acerca del proceso de vinculación en general y en particular en las carreras de ingeniería. Este análisis se orientó a determinar: las principales insuficiencias que existen en el proceso de vinculación en diferentes estados de la República Mexicana, sus potenciales no explotadas, la presencia de un enfoque integral en su concepción e implementación, así como los principales logros.

Además, la investigación se orientó a describir las experiencias obtenidas en estas carreras de la UANL de México, en cuanto a vías que pueden ser utilizadas para la formación de estudiantes a través del proceso de vinculación.

Se describen las acciones desarrolladas en las que participaron estudiantes de los últimos semestres de las 11 carreras de ingeniería, entre las que se encuentran: Ingeniería Mecánica Electricista, Ingeniería Mecánica Administradora, Ingeniería Administradora de Sistemas, Ingeniería en Electrónica y Comunicaciones, Ingeniería en Electrónica y Automatización, Ingeniería en Materiales, Ingeniería en Manufactura, Ingeniería en Meca trónica, Ingeniería en Aeronáutica, Ingeniería en Tecnología de Software e Ingeniería Biomédica.

En la facultad se desarrollan múltiples y variadas acciones para la vinculación con el entorno, para este estudio se han seleccionado aquellas que de manera más directa impactan en el desarrollo profesional del estudiantado en general y en su inserción en el mundo laboral una vez graduado. La descripción de las acciones se realiza tomando en cuenta sus características fundamentales, así como sus objetivos. Dicha caracterización constituye una vía de socialización de las diferentes posibles vías de concretar la vinculación de la universidad con el entorno con el fin de contribuir la formación de profesionales en ingeniería.

12 Jaime Arturo Castillo-Elizondo, Nivia Tomasa Álvarez-Aguilar y Arnulfo Treviño-Cubero

Los artículos de la Revista Electrónica Educare del Centro de Investigación y Docencia en Educación de la Universidad Nacional, Costa Rica, se comparten bajo términos de la Licencia Creative Commons: Reconocimiento, No Comercial, Sin Obra Derivada 3.0 Costa Rica. Las autorizaciones adicionales a las aquí delimitadas se pueden obtener en el correo: educare@una.cr 


\section{Resultados}

\section{Descripción de acciones para implementar la vinculación con base en experiencias de la UANL}

En el presente trabajo se presentan las acciones prácticas aplicadas correspondientes a una primera etapa de una investigación; aunque el proceso no ha concluido, se observan resultados positivos en particular en el conocimiento acerca de las perspectivas de trabajo y crecimiento profesional, posibilidades de superación que poseen las diferentes carreras, así como de las exigencias de los entes empleadores.

Los antecedentes de los 55 años de creada la Facultad de Ingeniería de la Universidad Autónoma de Nuevo León, que en la actualidad cuenta con 10 carreras, han aportado experiencias muy positivas que pudieran replicarse en escuelas y facultades de ingeniería tanto del país como de otros contextos.

No cabe duda [sic] que requerimos de un esfuerzo más sistemático y extendido de documentación de las experiencias institucionales de colaboración universidad-empresa, que permitan constituir un amplio banco de información en el que la vinculación adquiera sentido por sus propias realizaciones que por meras declaraciones retóricas. (IbarraColado, 2008, p. 324)

Se han implementado diferentes esquemas de vinculación tanto propios como los utilizados en otras facultades y escuelas, pero con innovación en su aplicación, también se han experimentado otras nuevas vías que permiten aportar a una gestión integral de este proceso.

Los nuevos esquemas en la vinculación se han visto favorecidos por diferentes procedimientos en cuanto a la colaboración con entes agrupadores de empresa y gobierno. En cada región donde están enclavadas las escuelas y facultades de ingeniería existen grandes potencialidades a la espera de ser utilizadas en función de una mayor eficiencia de la vinculación en cuanto a la formación profesional de los futuros grupos profesionales en ingeniería.

En la facultad se desarrollan múltiples acciones de vinculación no solo con empresas, sino también con otras instituciones sociales. Algunas de estas han sido descritas por Castillo (2016), en su trabajo presentado para ingresar en la Academia de Ingeniería de México sobre: La gestión de la vinculación en las facultades de ingeniería como vía para la formación de un ingeniero transformador de su entorno, entre las actividades referidas se encuentran: 
Encuentros de Vinculación, Congresos, Proyectos Escolares de Práctica Vinculada, Servicio Social, Prácticas Profesionales, Bolsa de trabajo, Reclutamientos en Campus, Exposiciones Laborales, Seguimiento de Egresados, Incubadora de Negocios, Proyectos de Investigación [e innovación], Servicios de Asesoría y Consultoría, Cursos de Capacitación y Educación Continua, [Talleres de reflexión para intercambio de experiencias entre empresarios, personal de la facultad y del gobierno], Parques de Innovación [Científica y] Tecnológica. (p. 17)

Además de las actividades enlistadas se pueden mencionar otras acciones que han surgido al tomar en cuenta tanto las exigencias sociales, como el acelerado desarrollo científico técnico. Entre estas se encuentran: desarrollo de proyectos conjuntos academia-empresa, implementación de la materia de "formación de personas emprendedoras", desarrollo de ferias de empleo, foros de entes empleadores, creación de la coordinación de responsabilidad social, entre otros.

A continuación se muestran las potencialidades para impactar en la formación profesional de la ingeniera y el ingeniero contemporáneos de algunas de las acciones mencionadas.

\section{Desarrollo de proyectos conjuntos academia- empresa}

Uno de estos proyectos en los que se han obtenido resultados positivos es el proyecto "La empresa en tu aula". Ha permitido insertar profesionales de las empresas en diferentes materias docentes en la facultad para que interactúen directamente con el estudiantado. Además del conocimiento que adquieren a través de las experiencias de profesorado-ente empresario, también se organizan visitas a las empresas con el propósito de obtener una mejor preparación. De esta forma, el estudiantado recibe una objetiva y real visión del funcionamiento y aplicación, en la industria, de las competencias que adquieren en su tiempo de estudios. Entre sus objetivos principales se encuentran:

- Enriquecer las asignaturas relacionadas con la especialidad de las diferentes carreras.

- Compartir experiencias prácticas en el aula.

- Preparar al estudiantado para su inserción laboral.

- Innovar en la educación de profesionales en ingeniería.

\section{Talleres de reflexión para intercambio de experiencias entre personas empresarias, personal de la facultad y del gobierno}

Estos talleres son de gran utilidad para concretar el enfoque integral de la gestión de la vinculación en función del desarrollo profesional del estudiantado. Se desarrollan con la presencia de representantes empresariales de la región, personal directivo y docente de la 
facultad y entes del gobierno. Tienen, como objetivo esencial, el intercambio de criterios y propuestas para lograr una vinculación más efectiva y sistemática. Posibilitan analizar de forma conjunta las áreas de oportunidad, las fortalezas, así como las perspectivas de vinculación de acuerdo con las condiciones y necesidades que presenten los diferentes actores que participan en la vinculación. Los acuerdos derivados del consenso de los participantes constituyen objetos de análisis y seguimiento y sirven de una constante retroalimentación.

\section{Creación de centros de innovación, investigación y desarrollo en ingeniería y tecnología}

Son centros multidisciplinarios de carácter integrador cuyo objetivo fundamental es generar y aplicar de manera innovadora conocimientos de frontera en las áreas de ingeniería y tecnología, entre las que pueden considerarse: nanotecnología, materiales avanzados, mecatrónica, ingeniería de software, entre otros.

Otro de los centros referidos por Castillo (2016) es el Centro de competitividady productividad en ingeniería (CCPI).

Este centro tiene como misión ofrecer servicios innovadores que involucran al gobierno, las empresas estatales, privadas y la universidad a través una colaboración donde cada uno de los sectores que participa obtenga beneficios. De esta forma constituye un polo de desarrollo científico y tecnológico para atender las problemáticas sociales. Se ha priorizado la construcción de este tipo de centro como una vía efectiva para estrechar la vinculación con el sector industrial, así como para la generación de proyectos de investigación y desarrollo socialmente responsables.

Además de obtener recursos que se revierten en el aspecto académico e investigativo de la facultad, también estos centros tienen como propósito contribuir al desarrollo profesional de estudiantes que a ellos se integran.

\section{Formación de personas emprendedoras}

Se desarrolla a través de una asignatura denominada "Formación de emprendedores" que posee un contenido dinámico que se adapta a los cambios del entorno empresarial. En el transcurso de ella el estudiantado realiza estudios de empresas internacionales y nacionales que son innovadoras, así como de modelos de negocios y de desarrollo tecnológico, con el fin de prepararse para la creación de su propia empresa.

Las clases se organizan por equipos de no más de 5 estudiantes, cada quien elige a una empresa para representarla. A través del semestre se conjugan los aspectos teóricos y prácticos con la elaboración de un proyecto denominado PIA (Proyecto integrador de aprendizaje). Dicho proyecto incluye un determinado modelo de negocios y el prototipo del proyecto ingenieril. 
doi: http://dx.doi.org/10.15359/ree.23-2.11

URL: http://www.una.ac.cr/educare

CORREO: educare@una.cr

Al culminar el semestre se elige el mejor proyecto por aula. Se seleccionan los mejores proyectos de los diferentes grupos para ser expuestos ante todo el estudiantado de la facultad y evaluados por un jurado integrado por sujetos empleadores de empresas importantes del entorno. Los mejores proyectos son premiados y presentados en diferentes foros estudiantiles. De este modo, se contribuye, no solo a la formación profesional, sino también al desarrollo de competencias para la investigación.

\section{Feria del empleo}

Este tipo de acción surge como una demanda tanto de la industria como de la facultad en cuanto a la necesidad de contribuir con la identificación de opciones de trabajo por parte del estudiantado lo que constituye un beneficio para este y también para las empresas.

Sus objetivos fundamentales se orientan al logro de una vinculación más efectiva con el sector empresarial, en particular, el sector privado con el estudiantado y personas egresadas. De este modo, se benefician las empresas por la posibilidad de cubrir sus vacantes en cualquiera de las siguientes modalidades: empleo, prácticas profesionales o servicio social.

El estudiantado puede encontrar en estas ferias diferentes opciones que no estaban a su alcance a través de otros medios. Una de sus principales ventajas es que pueden intercambiar con los grupos empleadores y tener una visión más completa de las ofertas laborales.

Entre sus mayores beneficios están: la inclusión de diversas empresas como entidades reclutadoras, la promoción de productos o servicios, el conocimiento por parte del estudiantado y el profesorado de las condiciones de trabajo y culturas de las diferentes empresas, así como el fortalecimiento de las relaciones entre entes empleadores, estudiantes y las coordinaciones de las diferentes carreras de ingeniería.

\section{Foro de entes empleadores}

Esta modalidad de vinculación juega una función muy importante, ya que ofrece retroalimentación a la facultad por parte de los grupos empleadores. Es de gran utilidad la información recabada acerca de las fortalezas y áreas de oportunidades que presenta el estudiantado en el tiempo de prácticas, así como el estudiantado egresado de acuerdo con las necesidades reales a las que se enfrentan en las empresas. Además, se exponen las necesidades y áreas de trabajo donde se requiere la prestación de servicios. Dichos foros se orientan a fortalecer tanto la formación de los futuros grupos profesionales en ingeniería como de las empresas. 


\section{Creación de la Subdirección de Responsabilidad Social}

Además de la Subdirección de Vinculación, se crea en el 2013 la Subdirección de Responsabilidad Social, como consecuencia del crecimiento de la facultad y la necesidad de realizar un mayor aporte al desarrollo del entorno involucrando a estudiantes y profesorado. Su propósito fundamental es potenciar los indicadores de las diferentes dimensiones que caracterizan una facultad socialmente responsable y a partir de ahí lograr un impacto positivo en los diferentes sectores sociales.

Una de las principales coordinaciones de esta subdirección orientadas al propósito mencionado es la de Servicio Social. Como bien indican García-Anciera, Castillo-Elizondo y Salinas-Reyna (2016, p. 3):

El Servicio Social es una de las principales conexiones que tiene la universidad con la sociedad, así mismo, da la apertura a que sus estudiantes de nivel superior de los programas educativos tengan una participación activa en la solución de diversos problemas que afecten su entorno.

Las acciones de la citada subdirección tienen un impacto positivo para el entorno, pues beneficia a varios grupos altamente vulnerables. De igual manera, al involucrar al estudiantado se potencia el desarrollo de competencias de carácter personal y profesional para un desempeño comprometido con la sociedad.

\section{Consideraciones finales}

Existen diversos estudios sobre el tema de la vinculación como problema prioritario en las universidades, su análisis permite concluir que encauzan este tema desde múltiples enfoques, criterios y posiciones. Se observa una coincidencia en cuanto a que este es un problema de importancia capital que requiere de constante renovación y perfeccionamiento y que, además, no puede centrarse solo en la obtención de recursos por parte de las instituciones educativas, ni tampoco en la satisfacción de las exigencias de los entes empleadores exclusivamente.

Los antecedentes del tema muestran área de oportunidades y potencialidades relacionadas con la satisfacción de los diferentes actores que participan en el proceso de vinculación. A su vez, existen insuficientes argumentos que deben ser tomados en cuenta no solo en la formación profesional del estudiantado, sino también en su desarrollo humano de manera compleja. Se detecta poca presencia del enfoque integral, lo que demanda analizar esta problemática con una visión más trascendente donde cada uno de los factores implicados realice sus aportaciones en aras del cumplimiento de los objetivos comunes. 
doi: http://dx.doi.org/10.15359/ree.23-2.11

URL: http://www.una.ac.cr/educare

CORREO: educare@una.cr

La práctica de la vinculación indica que los esquemas, vías y alternativas para su realización son más eficientes cuando se logra una sinergia entre todos los factores implicados. Dicha sinergia se asienta en una concientización de cada uno de ellos dirigida a promover una formación real, objetiva e integral de profesionales en ingeniería actual.

De acuerdo con lo antes expresado, la formación integral del estudiantado en ingeniería se concreta a través de las múltiples formas de su inclusión, en su tiempo de estudios, en actividades de diferente naturaleza. Los antecedentes de esta investigación y los fundamentos que la justifican, así como las acciones prácticas hicieron posible identificar una serie de principios que pudieran contribuir a llenar los vacíos existentes en los estudios del tema, entre estos se pueden enunciar:

1. Aplicación de un enfoque integral donde se involucren de manera proactiva todos los factores implicados en el proceso de la vinculación.

2. Potenciación del aspecto formativo del estudiantado de ingeniería conjuntamente con la obtención de recursos que se reviertan en crecimiento y transformación de las instalaciones de la facultad.

3. Concepción de la dimensión profesional en la formación del ingeniero y la ingeniera de un modo sistemático, y con una adecuada retroalimentación y control.

Los principios antes mencionados propiciarían que el estudiantado, al culminar sus estudios, esté mejor preparado con las habilidades y competencias necesarias para que al momento de egresar, cuente con un desarrollo profesional integral.

Las acciones basadas en las experiencias descritas en este artículo ponen de manifiesto las diversas y variadas opciones que pueden aplicarse en las facultades y escuelas de ingeniería para que la inserción del estudiantado en diferentes acciones potencie una formación profesional objetiva y prospectiva. Constituyen ejemplos de las potencialidades de las facultades de escuelas y facultades de ingeniería, para implementar variadas y múltiples vías y formas que pueden ser implementadas y adaptadas a diferentes contextos y que, a su vez, estimulan la búsqueda de otras novedosas e innovadoras que el propio desarrollo social y científico tecnológico exige.

\section{Referencias}

Alcántar, V. M. y Arcos, J. L. (2009). La vinculación como factor de imagen y posicionamiento de la Universidad Autónoma de Baja California, México, en su entorno social y productivo. Revista Electrónica de Investigación Educativa, 11(1), 1-20. Recuperado de https://redie. uabc.mx/redie/article/view/227/383 
Asociación Iberoamericana de Instituciones de Enseñanza de la Ingeniería (ASIBEI). (2015). ASIBEI Boletín. Recuperado de http://asibei.net/boletines/2015/agosto/

Asociación Iberoamericana de Instituciones de Enseñanza de la Ingeniería (ASIBEI). (2016). ASIBEI Boletín. Recuperado de http://asibei.net/boletines/2016/noviembre/

Bautista, E. G. (2014). La importancia de la vinculación universidad-empresa-gobierno en México. Revista Iberoamericana para la Investigación y el Desarrollo Educativo, 5(9), 107-127. Recuperado de https://www.ride.org.mx/index.php/RIDE/issue/view/1

Cámara-Zapata, J. M. (2016). Formación en ingeniería en España. IJERI: International Journal of Educational Research and Innovation, 5, 112-123. Recuperado de https://www.upo.es/ revistas/index.php/IJERI/article/view/1585/1321

Campos, G. y Sánchez, G. (2005). La vinculación universitaria: Ese oscuro objeto del deseo. Revista Electrónica de Investigación Educativa, 7(2), 1-13. Recuperado de https://redie.uabc. $\mathrm{mx} /$ redie/article/view/134

Cantú-Martínez, P. C. (2013). Las instituciones de educación superior y la responsabilidad social en el marco de la sustentabilidad. Revista Electrónica Educare, 17(3), 41-55. Recuperado de http://www.redalyc.org/articulo.oa?id=194128798004

Capote, G. E., Rizo, N. y Bravo, G. (2016). La formación de ingenieros en la actualidad. Una explicación necesaria. Universidad y Sociedad, 8(1), 21-28. Recuperado de http://scielo.sld. cu/pdf/rus/v8n1/rus03116.pdf

Castillo, J. A. (2016). La gestión de la vinculación en las facultades de ingeniería como vía para la formación de un ingeniero transformador de su entorno (Trabajo presentado para el ingreso a la Academia Mexicana de ingeniería). Recuperado de https://es.slideshare.net/ AcademiaDelngenieriaMx/la-gestin-de-la-vinculacin-en-las-facultades-de-ingenieracomo-va-para-la-formacin-de-un-ingeniero-transformador-de-su-entorno

Castillo, J. A., Reynoso, M., Álvarez, N. y Torres, A. (2013). La vinculación y la formación integral del estudiante de ingeniería. México: Pearson.

Centro de Investigación para el Desarrollo (CIDAC). (2014). Encuesta de competencias profesionales ¿Qué buscan -y no encuentran- las empresas en los profesionistas jóvenes? México: Autor. Recuperado de http://cidac.org/esp/uploads/1/encuesta competencias profesionales 270214.pdf

D'Este, P., Castro, E y Molas-Gallart, J. (2014). Documento de base para un "Manual de indicadores de vinculación de la universidad con el entorno socioeconómico" (Manual de Valencia). Valencia: CSIC-UPV, Instituto de Gestión de la Innovación y del Conocimiento. Recuperado de http://hdl.handle.net/10261/132865 
doi: http://dx.doi.org/10.15359/ree.23-2.11

URL: http://www.una.ac.cr/educare

CORREO: educare@una.cr

Fernández, J. A. (2001). Elementos que consolidan el concepto de profesión. Notas para su reflexión. Revista Electrónica de Investigación Educativa, 3(2), 23-39. Recuperado de http:// redie.uabc.mx/redie/article/view/40

García-Anciera, C., Castillo-Elizondo, J. A. y Salinas-Reyna, I. M. (2016). Seguimiento al servicio social del estudiantado de ingeniería a través de la plataforma Nexus. Revista Electrónica Educare, 20(2), 1-23. doi: https://doi.org/10.15359/ree.20-2.15

García, C., Carreón, J. y Hernández, J. (2014). La formación profesional de capital humano en la civilización del cambio climático. Revista Internacional de Investigación en Ciencias Sociales, 10(1), 107-125. Recuperado de http://revistacientifica.uaa.edu.py/index.php/riics/article/ view/201/189

García J. (2004). Estudios descriptivos. Nure Investigación, 7, 1-3. Recuperado de http//www. nureinvestigacion.es/OJS/index.php/nure/article/view/180

Gutiérrez, N. G. (2004). La vinculación en el ámbito científico-tecnológico de México:Instituciones de educación superior en interacción con distintos actores. Revista Latinoamericana de Estudios Educativos, 34(2), 47-94. Recuperado de http://www.redalyc.org/articulo. oa?id=27034204

Herrera, J. L. (2006). La formación de los profesionales universitarios en las empresas en el contexto dubano. Revista Electrónica de la Red de Investigación Educativa, 1(4), 1-18. Recuperado de http://revista.iered.org/actual/pdf/jlherrera.pdf

Ibarra-Colado, E. (2008). Regulación social de la 'triple hélice' en América Latina: Diálogos en busca de un proyecto distinto. Reseña del libro La vinculación universidad-empresa: miradas críticas desde la universidad pública, por S. Llomovatte (Dir.). Revista Mexicana de Investigación Educativa, 13(36), 319-327. Recuperado de http://www.redalyc.org/articulo. oa?id=14003616

Jiménez-Montecinos, A. (2016). Relaciones universidad-empresa: Hacia una productividad basada en innovación. Revista Gestióny Tendencias, 1(2),7-10. doi:https://doi.org/10.11565/ gesten.v2i1.11

Kindelán, M. P. y Martín A. M. (2008). Ingenieros del siglo XXI: Importancia de la comunicación y de la formación estratégica en la doble esfera educativa y profesional del ingeniero. Arbor, Ciencia, Pensamiento y Cultura, 184(732) 731-742. Recuperado de https://doi.org/10.3989/ arbor.2008.i732.218

López, J. (2014). Modelos interpretativos de la relación estado-empresa-universidad. Clío América, 8(15), 111-122. doi: https://doi.org/10.21676/23897848.833 
López-Parada, C E. y Martínez-Dajui, E. (2014). Esquema de vinculación de las universidades tecnológicas del Estado de puebla en la formación de estudiantes. Ra Ximhai, 10(3), 1-13. Recuperado de http://www.redalyc.org/pdf/461/46131111001.pdf

Pacheco, T. (2012). La formación profesional: Práctica institucionalizada, estrategia escolarizada y proyecto educativo en permanente evaluación. Revista Iberoamericana de Educación, 60(3), 1-10. Recuperado de https://rieoei.org/RIE/article/view/1304

Palma, C. A. (2012). Nuevos retos para el ingeniero en el siglo XXI. Revista Ingeniería e Innovación, 4, 61-65. Recuperado de http://www.redicces.org.sv/jspui/bitstream/10972/1973/1/4nuevos-retos-para-el-ingeniero-en-el-siglo-xxi.pdf

Pérez, A. (2016). Razones que motivan la vinculación de la universidad con la empresa: Análisis comparativo México y Argentina. Revista RAITES, 2(4), 10-28. Recuperado de http://www. itc.mx/ojs/index.php/raites/article/view/517/646

Pertuz, R. (2014). Modelo de estructura organizacional para los institutos universitarios, vinculante con la realidad socioeconómica venezolana. Revista Electrónica Educare, 18(1), 97-117. Recuperado de http://www.revistas.una.ac.cr/index.php/EDUCARE/article/ view/5565/5398

Sarabia-Altamirano, G. (2016). La vinculación universidad-empresa y sus canales de interacción desde la perspectiva de la academia, de la empresa y de las políticas públicas. CienciaUAT, 10(2), 13-22. Recuperado de https://doi.org/10.29059/cienciauat.v10i2.741

Soto, R., Castaños, H., García, O., Parra, P., Espinosa, J. y Vázquez, J. L. (2007). Vinculación universidad-empresa-estado en la realidad actual de la industria farmacéutica mexicana. Edusfarm, revista d'educació superior en Farmàcia, 2, 1-27. Recuperado de http://www. publicacions.ub.edu/revistes/edusfarm2/documentos/125.pdf

Valera, R. (2010). El proceso de formación del profesional en la educación superior basado en competencias: El desafío de su calidad, en busca de una mayor integralidad de los egresados. Civiliza. Ciencias Sociales y Humanas, 10(18), 117-133. Recuperado de http:// www.redalyc.org/articulo.oa?id=100220339010 